\title{
Many-body interactions and high-pressure equations of state in rare-gas solids
}

\author{
Yu.A. Freiman and S.M. Tretyak \\ B. Verkin Institute for Low Temperature Physics and Engineering of the National Academy of Sciences of Ukraine \\ 47 Lenin Ave., Kharkov 61103, Ukraine
}

Received December 18, 2006

\begin{abstract}
The $T=0 \mathrm{~K}$ equations of state (EOS) of rare-gas solids (RGS) ( $\mathrm{He}, \mathrm{Ne}, \mathrm{Ar}, \mathrm{Kr}$, and $\mathrm{Xe}$ ) are calculated in the experimentally studied ranges of pressures accounting for two- and three-body interatomic forces. Solid-state corrections to the pure two-body Aziz et al. potentials included the long-range Axilrod-Teller three-body interaction and short-range three-body exchange interaction. The energy-scale and length-scale parameters of the latter were taken as adjustable parameters of theory. The calculated $T=0 \mathrm{~K}$ EOS for all RGS are in excellent agreement with experiment in the whole range of pressures. The calculated EOS for Ar, $\mathrm{Kr}$, and Xe exhibit inflection points where the isothermal bulk moduli have non-physical maxima indicating that account of only three-body forces becomes insufficient. These points lie at pressures 250, 200, and 175 $\mathrm{GPa}$ (volume compressions of approximately 4.8, 4.1, and 3.6) for Ar, Kr, and Xe, respectively. No such points were found in the calculated EOS of $\mathrm{He}$ and $\mathrm{Ne}$. The relative magnitude of the three-body contribution to the ground-state energy with respect to the two-body one as a function of the volume compression was found to be non-monotonic in the sequence $\mathrm{Ne}-\mathrm{Ar}-\mathrm{Kr}-\mathrm{Xe}$. In a large range of compressions, $\mathrm{Kr}$ has the highest value of this ratio. This anomally high three-body exchange forces contributes to the EOS so large negative pressure that the EOS for $\mathrm{Kr}$ and $\mathrm{Ar}$ as a function of compression nearly coincide. At compressions higher approximately 3.5, the curves intersect and further on the EOS of Kr lies lower than that of Ar.
\end{abstract}

PACS: 64.60.Cn Order-disorder transformations; statistical mechanics of model systems; 67.80. - s Solid helium and related quantum crystals;

67.90.+z Other topics in quantum fluids and solids; liquid and solid helium.

Keywords: rare-gas solids, equations of state, two and tree-body interatomic forces.

\section{Introduction}

The availability of equation of state (EOS) and structure measurements in the megabar pressure range imparted a new momentum to the problem of the application of the many-body potentials to the analysis of the experimental results [1-8]. It is well-known [9] that the potential consisting from the pure two-body potential and the long-range Axilrod-Teller (AT) three-body correction makes it possible to reproduce the zero-temperature zero-pressure properties and low-pressure EOS data of solid $\mathrm{Ne}, \mathrm{Ar}, \mathrm{Kr}$, and $\mathrm{Xe}$. As known, the AT forces is a three-body analogue of the London two-body dispersion forces. Loubeyre [6,7] calculated the EOS of solid $\mathrm{He}$, Ar, $\mathrm{Kr}$, and $\mathrm{Ne}$ by using the potential which included also the three-body exchange correction term and obtained good agreement with experiment. This term originates from the Pauli exclusion principle and means that the charge distributions of two atoms change in the presence of a third atom. Kim et al. [8] represented the exact three-body exchange interaction by a short-range pair correction term and found the adjusting parameters of this term from the condition that it corrects deviations between experimental pressures and pressures calculated without this term. The resulting EOS for solid $\mathrm{Kr}$ and $\mathrm{Xe}$ were in fair agreement with experiment to the highest experimental pressures. Thus it has been demonstrated that the EOS of these systems may be well described using the effective two-body potential with a specially adjusted short-range correcting term instead of the real three-body exchange interaction. Subsequently, this approach was used to describe materials other than RGS, for example, in the case of compressed solid hydrogen the short-range behavior of the Silvera-Goldman potential [10] was corrected by Hemley et al. [11] with a help of the same two-term polinomial correcting term. Thus EOS calculations are generally believed to be not very sensitive to the difference between the many-body 
potential and the effective two-body one. At the same time, the structural implications of these two types of potentials may be different $[1,3]$. As was shown in Refs. 1 and 3, the lattice distortion parameter in compressed solid helium and hydrogen is very sensitive to the real nature of the intermolecular potential and thus can be used as a probe of the many-body forces. The same is true about other directional characteristics, for example, elastic moduli. Nontheless solid-state calculations based on many-body potentials are scarce. Not much is known about the role of many-body forces in such high-pressure phenomena as high-pressure polymorphic transitions, pressure-driven metallization, pressure-induced dissociation, etc. Many issues concerning the EOS and phase diagrams of RGS remain still to be understood.

In this article the $T=0 \mathrm{~K}$ EOS of $\mathrm{He}, \mathrm{Ne}, \mathrm{Ar}, \mathrm{Kr}$, and $\mathrm{Xe}$ are calculated in the experimentally studied ranges of pressures accounting for two- and three-body interatomic forces. Solid-state corrections to the pure two-body Aziz et al. potentials included the long-range Axilrod-Teller three-body interaction and short-range three-body exchange interaction. Following Loubeyre [6,7], we took the latter for all the RGS family in a Slater-Kirkwood form. The main differences of our approach from that of Refs. 6 and 7 are the following: (a) we took into account zero-point contribution of the many-body forces to the free energy of the systems; (b) we took into account the contribution of the three-body exchange interaction from all the different triplets formed by two neighbors of a central atom lying inside the sphere of five nearest neighbor distances; (c) the energy-scale and length-scale parameters of the three-body exchange interaction were taken as adjustable parameters of theory. The calculated $T=0 \mathrm{~K}$ EOS for all RGS are in excellent agreement with experiment in the whole range of pressures.

\section{Intermolecular potential}

The best available pair potentials for rare gas solids are the Aziz pair potentials ( $\mathrm{He})$ [12], (Ne) [13], ( $\mathrm{Ar}$ and $\mathrm{Kr}$ ) [14], (Xe) [15], which reproduce a variety of experimental gas phase data. as well as zero-temperature, zero-pressure properties of solid phase. These potentials can be represented in the form of the Silvera-Goldman potential [10]

$$
U_{p}(R)=\exp \left(\alpha-\beta R-\gamma R^{2}\right)-f_{c}(R) \sum_{6,8,10} C_{n} R^{-n} .
$$

Here $R$ is the interatomic distance, $\alpha, \beta, \gamma$ are coefficients of the exponential repulsive term, $C_{n}$ are the dispersion coefficients. $f_{c}(R)$ is a damping or attenuation function that smoothly joints the multipole terms with the short-range exponential repulsion term. It prevents from singularity at the origin by the long-range multipole terms.

$$
f_{c}(R)=\left\{\begin{array}{lr}
\exp \left[-\left(R^{*} / R-1\right)^{2}\right], & R<R^{*}, \\
1, & R>R^{*},
\end{array}\right.
$$

where $R^{*}=D R_{m}, R_{m}$ is the position of the potential minimum of the potential. Parameters of the Aziz potentials (in a.u.) are given in Table 1.

However, the pure pair potential does not describe properly the properties of solid-state phase and manybody corrections should be taken into account. At small pressures the main correction comes from the three-body dispersion interaction. At high pressures the many-body exchange effects should be taken into account. The equation-of-state-calculations based on the Aziz pair potentials give pressures which are systematically above the experimental equation of state; this illustrates the fact that at short distances the Aziz potential is too stiff and should be soften by adding the three-body exchange correction term. The short-range correction to the pair potentials originates from alteration of the charge clouds of two molecules in the presence of neighboring molecules due to the Pauli exclusion principle. Ab initio calculations of Bulski and Chalasinski [16] showed that the contribution of the three-body overlap potentials are important for rare-gas atoms. Here we restrict ourselves to the three-particle interaction, which consists of three-body dispertion forces (Axilrod-Teller forces) and tree-body exchange forces.

Table 1. Parameters (in a.u.) of pair potential

\begin{tabular}{c|c|c|c|c|c|c|c|c}
\hline \hline Substance & $\alpha$ & $\beta$ & $\gamma \cdot 10^{3}$ & $C_{6}$ & $C_{8}$ & $C_{10}$ & $D$ & $R_{m}$ \\
\hline \hline $\mathrm{He}[12]$ & 2.925 & 2.381405 & 0 & 1.45995 & 14.21950 & 187.1926 & 1.28 & 5.4379 \\
$\mathrm{~N} 2[13]$ & 4.786192 & 2.373569 & 3.808392 & 6.44696 & 96.4992 & 1519.985 & 1.36 & 5.841140 \\
$\operatorname{Ar}[14]$ & 4.630839 & 1.518400 & 35.961866 & 64.2995 & 1639.9865 & 50999.49 & 1.36 & 7.0987520 \\
$\operatorname{Kr}[14]$ & 4.251076 & 1.240412 & 40.54818 & 130.89515 & 3719.97 & 166938.3 & 1.28 & 7.574018 \\
$\operatorname{Xe}[15]$ & 2.93519 & 0.65698 & 72.8075 & 288.758 & 10999.9 & $5.6 \cdot 10^{5}$ & 1.45 & 8.24430 \\
\hline \hline
\end{tabular}


Let three paticles form a triangle with sides $r_{1}, r_{2}, r_{3}$ and angles $\phi_{1}, \phi_{2}, \phi_{3}$. Then the total three-body potential $U_{\text {tr }}$ is given by a Slater-Kirkwood-type expression $[17,6]$

$$
\begin{aligned}
U_{\mathrm{tr}}=\{ & \left.-A \exp \left[-\alpha_{\mathrm{tr}}\left(r_{1}+r_{2}+r_{3}\right)\right]+\frac{C_{\mathrm{tr}}}{r_{1}^{3} r_{2}^{3} r_{3}^{3}}\right\} \times \\
& \times\left(1+3 \cos \phi_{1} \cos \phi_{2} \cos \phi_{3}\right) .
\end{aligned}
$$

Table 2. Parameters (in a.u.) of three-body potential (Eq. 3)

\begin{tabular}{c|c|c|c}
\hline \hline Substance & $A$ & $\alpha_{\mathrm{tr}}$ & $C_{\mathrm{tr}}$ \\
\hline \hline $\mathrm{He}$ & $30.644^{a}$ & $1.0245^{a}$ & $1.4807^{a}$ \\
$\mathrm{Ne}$ & $566.969^{b}$ & $1.1896^{b}$ & $11.835^{d}$ \\
$\mathrm{Ar}$ & $1331.369^{c}$ & $0.905888^{c}$ & $526.5^{d}$ \\
$\mathrm{Kr}$ & $1717.154^{c}$ & $0.842728^{c}$ & $1578,3^{d}$ \\
$\mathrm{Xe}$ & $1964.058^{c}$ & $0.770203^{c}$ & $5283^{d}$ \\
\hline \hline
\end{tabular}

Notes: ${ }^{a}$ Ref. $6 ;{ }^{b}$ this work (slightly modified parameters from Ref. 4); $c_{\text {this }}$ work (slightly modified parameters from Ref. 7, see the text); ${ }^{d}$ Ref. 18 .

The first exponential term of this equation represents the exchange three-body interaction which at small interatomic distances descibes the alterations of the charge densities of two interacting molecules by the presence of a third one. The second term, known as the Axilrod-Teller one, is the large-distance asymptote of the triple-dipole interaction. At large interatomic separations, the main contribution to the $U_{\text {tr }}$ term comes from the Axilrod-Teller term and at smaller internuclear separations the three-body exchange overlap interaction is the dominant term. Parameters of the threebody potential (in a. u.) are given in Table 2.

It could be argued about the need of taking into account of the three-body interactions since an effective pair potential which includes their isotropic average can well reproduce the experimental equation of state. However the effective pair potential which best reproduces the equation of state worst reproduces the elastic constants, which are direction-dependent quantities. But what is more important, the three-body forces and the effective pair forces are acting differently when considering such phenomena as fcc-hcp phase transition, and pressure-driven metallization and molecular-nonmolecular transitions. In the case of hop simple solids $\left(\mathrm{He}\right.$ or $\left.\mathrm{H}_{2}\right)$ the effective two-body potential and a real one, consisting of a sum of the pair and three-body terms foretold qualitatively different pressure dependence of the $c / a$ ratio [3].

\section{3. $T=0 \mathrm{~K}$. Equation of states}

To use the low- and room-temperature measurements in inferring correct magnitudes of the three-body interaction it is convenient to reduce all the experimental data to $0 \mathrm{~K}$. To this end we will extract the thermal pressure $P_{\text {th }}$ from from available experimental data. This term was calculated by the use of the Mie-Grüneisen model

$$
P_{\text {th }}=(3 R T \gamma / V) / D(\Theta / \mathrm{T}),
$$

where $R$ is the gas constant, $D(x)$ is the Debye function, $\Theta$ is the Debye temperature, and $\gamma$, the Grüneisen parameter, is the logarithmic volume derivative of $\Theta$ :

$$
\gamma=-d \ln \Theta / d \ln V
$$

By integrating the latter equation with a specific form for the volume dependence of the Grüneisen constant we obtain an explicit dependence of the Debye temperature on volume. Analyzing different models, Holt and Ross [19] concluded that the linear approximation

$$
\gamma=\gamma_{0}+\gamma_{1}\left(V / V_{0}\right)
$$

where $V_{0}$ is the zero-pressure volume, is appropriative for solids such as the RGS for which the closed shell repulsive forces determine the EOS at high compressions. They suggested that $\gamma_{0}$ is approximately 0.5 . As a result, we get

$$
\Theta / \Theta_{0}=\sqrt{V_{0} / V} \exp \left[\gamma_{1}\left(1-V / V_{0}\right)\right] .
$$

Using the described procedure, we obtain $P_{0 \mathrm{~K}}$ by substracting the thermal contribution from experimental pressures. In these calculations the following parameters were used: Ne: $\gamma_{0}=0.5, \gamma_{1}=2.05$ [20]; Kr: $\gamma_{0}=0.5$, $\gamma_{1}=2.3$ [8]; Xe: $\gamma_{0}=0.5, \gamma_{1}=2.4$ [21]. Ross et al. [22] calculated the dependence $\gamma(V)$ for solid Ar. Approximating their $\gamma(V)$ by the linear function Eq. (6) we obtained $\gamma_{1}=1.416, \gamma_{0}=1.066$.

The ground-state energy $E_{g s}$ of the system can be written in the form

$$
E_{g s}=U_{p}+U_{\mathrm{tr}}+U_{z p},
$$

where $U_{z p}$ is the energy of zero-point oscillations.

In the following, the pressure region where zero-point effects play a decisive role will be excluded from consideration, so in calculations of the zero-point contribution standart methods of lattice dynamics have been used, in particular, the Einstein approximation. In this approximation the zero-point energy can be found in the following way:

$$
U_{z p}=\frac{\hbar}{2}\left(\omega_{x}+\omega_{y}+\omega_{z}\right),
$$

where $\omega_{\alpha}(\alpha=x, y, z)$ are frequences of the harmonic oscillations of helium atoms near their equilibrium positions. 


\subsection{Solid $\mathrm{He}$}

An accurate determination of the EOS of ${ }^{4} \mathrm{He}$ has been an important research objective for decades [2,23-29]. Using techniques originally developed for high-pressure $\mathrm{x}$-ray diffraction of solid hydrogen, the crystal structure of solid helium was determined by single-crystal synchrotron methods up to $23 \mathrm{GPa}$ at $300 \mathrm{~K}$ [27] and demonstrated that solid helium has hexagonal-closed-packed structure over this $P-T$ range, in contrast to theoretical predictions. The highest compression reached in the EOS experiments is 8.4 .

The calculated dependence of $P(V)$ for solid helium is shown in Fig. 1 along with the experimental results from Refs. 23,27,29. It is seen that the pair potential is too stiff and overestimates the pressure values calculated with its help considerably. The negative contribution from the three-body interaction becomes appreciable starting approximately at three-fold compression and increases rapidly with pressure. At pressures over approximately 5 $\mathrm{GPa}$ (four-fold compression), the pair interaction approximation becomes inadequate. The dotted curve at the same Figure shows the pressure values calculated disregarding the contribution from zero-point oscillations. It is seen that the effect of the zero-point motion remains substantial up to the highest pressure reached, though it gradually decreases with rising pressure.

The pressure of solid helium on the logarithmic scale as a function of the relative volume change $V / V_{0}$ is shown in the Inset in Fig. 1,a. The EOS in this form readily illustrates limiting pressures or compressions where the approximation, which takes into account twoand three-body interactions and neglects more-body (four-body first of all) interactions, fails. As we will see below, a signature of such inedequacy is an inflection point, which can be seen at the high-pressure region of the EOS on the logarithmic scale of Ar, Kr, and Xe. The inflection point at the EOS means that there is a non-physical maximum at the derivative $\partial P / \partial V$, i.e., at the isothermal bulk modulus $B_{T}(V)$. In the case of helium, in the studied region ( $P$ up to $60 \mathrm{GPa}$ or near ten-fold compressions) no inflection point was found at the curve $\ln P\left(V / V_{0}\right)$, which we consider as evidence that we are still within the limits of the adopted approximation.

The contributions of the pair and triple forces, and zero-point oscillations to the ground-state energy of solid $\mathrm{He}$ are shown in Fig 1,b. As can be seen, the relationship between the contributions is rather complicated and is essentially different in different regions of the molar volume. At relatively small compressions $V_{0} / V \lesssim 3$, the ground-state energy $E_{g s}$ is dominated by the contribution of zero-point oscillations $E_{z p}$. At $V_{0} / V \approx 3$ the contributions of the pair and triple forces compensate each other $E_{p}+E_{\mathrm{tr}}=0$ and thus $E_{g s}=E_{z p}$. In the range of compressions $3 \lesssim V_{0} / V \lesssim 4$ the inequality holds $E_{g s}>E_{z p}>E_{p}$. At $V \sim 3.5 \mathrm{~cm}^{3} / \mathrm{mol}$
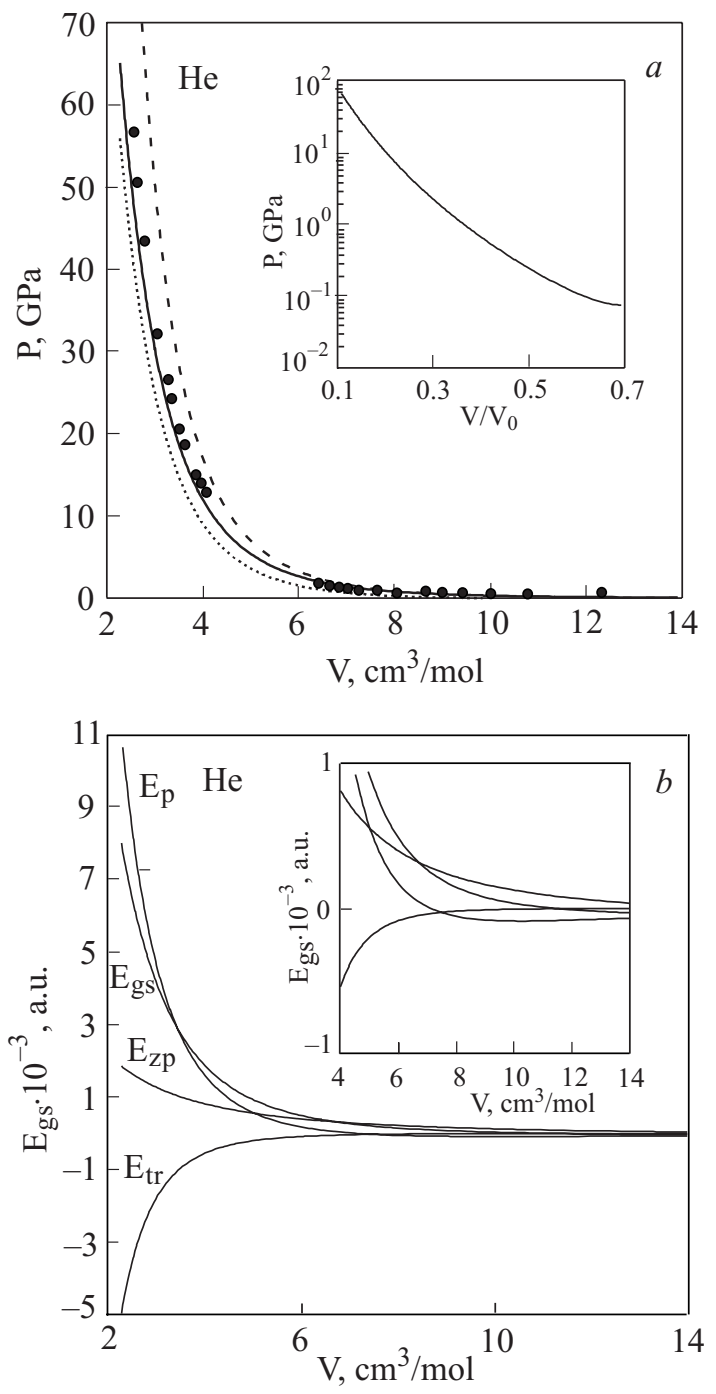

Fig. 1. The calculated $T=0 \mathrm{~K}$ equation of state for solid He. Solid symbols are experimental data from Refs. 23,27,29. Solid curve was calculated taking into account the pair Aziz potential, three-body potential, and zero-point contribution, dashed curve was obtained disregarding the three-body potential; dotted curve was obtained without regard for the zero-point oscillations. The inset shows the zero-temperature pressures on the logarithmic scale as a function of compression $(a)$. Contributions of the pair and triple forces, and zero-point oscillations to the ground-state energy $(b)$.

(six-fold compression), $E_{z p}+E_{\mathrm{tr}}=0$ and $E_{g s}=E_{p}$. At still higher compresions the static lattice contributions $E_{p}, E_{\mathrm{tr}}$ dominate $E_{z p}$ and consequently the EOS.

\subsection{Solid $\mathrm{Ne}$}

The behavior of neon at high densities has been the subject of comparatively few experimental and theoretical investigations in relation to studies of other rare-gas solids. Early high-pressure measurements of the heavy RGS by Anderson and Swenson [30] by using the piston-displacement technique provided low-temperature EOS data for solid neon up to $2.1 \mathrm{GPa}$. Diamond-an- 
vil-cell studies by Hazen et al. [31] showed that at room temperature fluid neon freezes at $14.4 \mathrm{GPa}$. Finger et al. [32] in single-crystal x-ray diffraction studies determined the crystal structure and equation of state at room temperatures from the freezing presure up to $14.4 \mathrm{GPa}$. It was shown that solid neon retains the cubic closed-packed structure. Hemley et al. [20] using energy-dispersive synchrotron $x$-ray diffraction techniques investigated the crystal structure and equation of state up to $110 \mathrm{GPa}$ at room temperatures. They showed that solid neon remains an insulator with the fcc structure in this $P-T$ range. The highest volume compression reached in experiment is 3.56 , more than two times less than for solid helium.

Hemley et al. $[20,33]$ compared their experimental data with results of their own lattice dynamics calculations using several different pair potentials. As it usually occurs with the EOS calculated with pure two-body potentials, the EOS calculated with the Aziz-Slaman potential [13] exhibits good agreement with experiment at low pressure, but at higher pressures the calculated pressures are significantly overestimated (see also comments to this point in Ref. 4). On the other hand, an effective exp-6 potential can be found that gives a good fit at the high-pressure region at the sacrifice of agreement at low compressions.

Path-integral Monte Carlo simulations of solid neon [4] confirmed inadequacy of pure two-body potentials for the high-pressure range. The inclusion of the three-body interaction in the Slater-Kirkwood form with two adjustible parameters $\left(A\right.$ and $\left.\alpha_{\text {tr }}\right)$ brought the results of simulations into a good agreement with the experimental results. Our calculations with the intermolecular potential consisting of the Aziz-Slaman pair potential [13] plus the three-body interaction with the parameters of the threebody short-range term from Ref. 4 ( $A=566.94$ a.u., $\alpha_{\mathrm{tr}}=$ $=1.1636$ a.u.) confirm results of the simulation. Using slightly modified parameters (see Table 2) we obtained excellent agreement with the experimental results in all the studied range of pressures (Fig. 2).

There were several theoretical predictions for behavior at much higher densities [34-37]. In the high-pressure region, the Birch-Murnaghan EOS based on the experimental results agrees well with the pressures calculated by Boettger and Trickey [36] and by Boettger [37] whereas the pressures calculated by Zharkov and Trubitsyn [34] and by Hama [35] are systematically lower. Our calculation for the terapascal range is given in the Inset in Fig. 2,a. As in the case of solid helium, no inflection point was found in the high-pressure range implying that we did not fall outside the limits of application of the adopted approximation. As show the comparison with the theoretical results for the terapascal range, our results are in excellent agreement both with the Birch-Murnaghan EOS based on the experimental results of Hemley et al. [20] and that of Refs. 36 and 37.
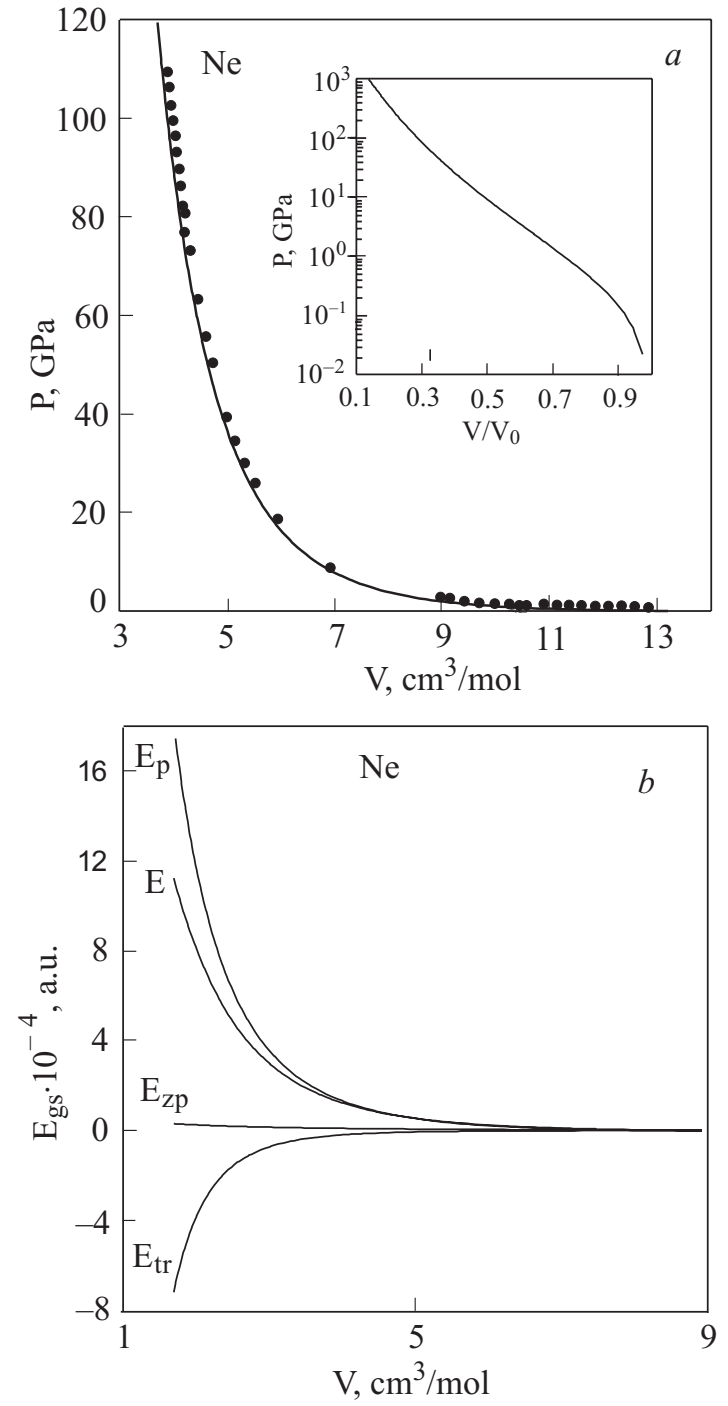

Fig. 2. The calculated $T=0 \mathrm{~K}$ equation of state for solid Ne. Solid symbols are experimental data: low pressure data are from Ref. 30, high-pressure data are from Ref. 20. The inset shows the zero-temperature pressures on the logarithmic scale extended to the terapascal range as a function of compression $(a)$. Contributions of the pair and triple forces, and zero-point oscillations to the ground-state energy $(b)$.

The contributions of the pair and triple forces, and zeropoint oscillations to the ground-state energy for solid $\mathrm{Ne}$ are shown in Fig 2,b. This figure is typical for the heavy RGS (Ne, $\mathrm{Ar}, \mathrm{Kr}, \mathrm{Xe})$ and diverges considerably from that for solid $\mathrm{He}$. At the low compressions $\left(V_{0} / V\right.$ higher $\left.\gtrsim 1.5\right)$, the ground state $E_{g s}$ and consequently the EOS is dominated by the static lattice contributions $E_{p}, E_{\mathrm{tr}}$. At the highest compression ( $V \approx 3.5 \mathrm{~cm}^{3} / \mathrm{mol}$ ) the zero-point contribution to the ground state of $\mathrm{Ne}$ is about $10 \%$. The figures for $\mathrm{Ne}, \mathrm{Ar}, \mathrm{Kr}$, and $\mathrm{Xe}$ look alike except that the respective zero point contributions to the ground-state energy for a given $V / V_{0}$ are progressively go down as one passes from $\mathrm{Ne}$ to $\mathrm{Xe}$. 


\subsection{Solid Ar, $\mathrm{Kr}$, and $\mathrm{Xe}$}

The low-pressure low-temperature piston-displacement measurements by Anderson and Swenson [30] gave EOS of solid argon to pressures of $2.0 \mathrm{GPa}$. Room temperature high-pressure measurements of Ross et al. [22] extended the EOS data to pressures of $80 \mathrm{GPa}$. The highest volume compression reached in experiment is 2.83 , near three times less than in solid helium.

The calculated $T=0$ dependence of $P(V)$ for solid argon is shown in Fig. 3 along with the experimental results. In our calculations we used slightly modified parameters $A$ and $\alpha_{\text {tr }}$ of the short-range three-body potential (see Table 2 ) compared to that from Ref. 7 ( $A=1328.67$ a.u., $\alpha_{\text {tr }}=0.87314$ a.u.). Agreement between theory and experiment is excellent both in the low- and high-pressure range. Our calculation extended for the terapascal range is given in the Inset in Fig. 3. In contrast to solid helium and neon, there is an inflection point at the $P(V)$ curve for Ar at $V / V_{0} \approx 0.21(P \approx 250 \mathrm{GPa}) . \mathrm{Kr}$ and $\mathrm{Xe}$ also exhibit analogous inflection points at their EOS (see Insets in Figs. 4 and 5$)$ at $V / V_{0} \approx 0.245$ and $0.28(P \approx 200 \mathrm{GPa}$ and $175 \mathrm{GPa}$ ) respectively. As was said above, at this points the isothermal bulk modulus $B_{T}(V)$ has a non-physical maximum indicating that the adopted approximation taking into account pair- and triple-body forces fails.

In addition to low-pressure data of Anderson and Swenson [30] there are two sets of high-pressure data for solid krypton. EOS measurements by Polian et al. [38,39] up to $30 \mathrm{GPa}$ were performed by $\mathrm{x}$-ray energy-dispersive techniques using synchrotron radiation and the elastic properties were determined using Brillouin scattering. $\mathrm{X}$-ray measurements by Aleksandrov et al. [40] gave the EOS data up to $52 \mathrm{GPa}$. The highest volume compression

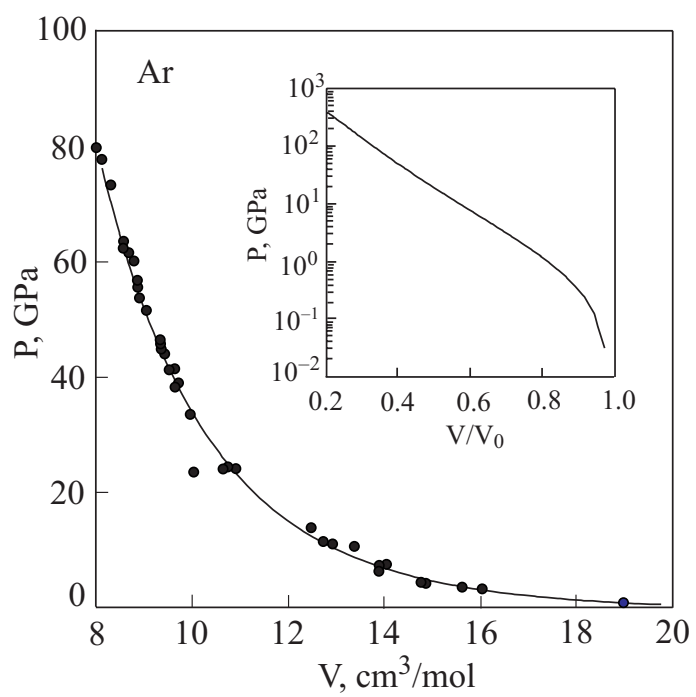

Fig. 3. The calculated $T=0 \mathrm{~K}$ equation of state for solid Ar. Solid symbols are experimental data of Ross et al. [22]. The inset shows the zero-temperature pressures on the logarithmic scale extended to the terapascal range as a function of compression. reached in experiment is 2.375 , the lowest value among the RGS solids.

The calculated zero-temperature dependence of $P(V)$ for solid krypton is shown in Fig. 4 along with the experimental results. In our calculations parameters $A$ and $\alpha_{\text {tr }}$ of the short-range three-body potential were slightly modified (see our values in Table 2) compared to that from Ref. 7 ( $A=1717.19$ a.u., $\alpha_{\text {tr }}=0.81811$ a.u.). As can be seen from Fig. 4, except two uppermost experimental points at 52 and $47.5 \mathrm{GPa}$, which most likely are in error, agreement between our calculations and all available experimental results (including low-pressure results by Anderson and Swenson not shown in Figure) are excellent. Our calculation extended for the terapascal range is given in the Inset in Fig. 4.

The EOS of kripton was calculated in Refs. 7,8, and 41. Loubeyre [7] using the self-consistent phonon approximation and Barker [41] using Monte Carlo method on the base of the same pair plus three-body intermolecular potential obtained that the pressure is somewhat underestimated compared to experiment. On this ground Barker concluded that the three-body exchange term is too high and that the best agreement with experiment can be obtained using the Axilrod-Teller interaction as the only many-body interaction. This conclusion is in variance with results of statistical mechanical calculations by Kim et al. [8]. They introduced an effective two-term polinomial short-range term to the pair plus Axilrod-Teller potential and found parameters of this term from the condition that it minimizes the deviation between theory and experiment. The resulted EOS is in fair agreement between theory and experiment to the highest experimental pressures. In fact, we found parameters of the short-range exchange term from the same condi-

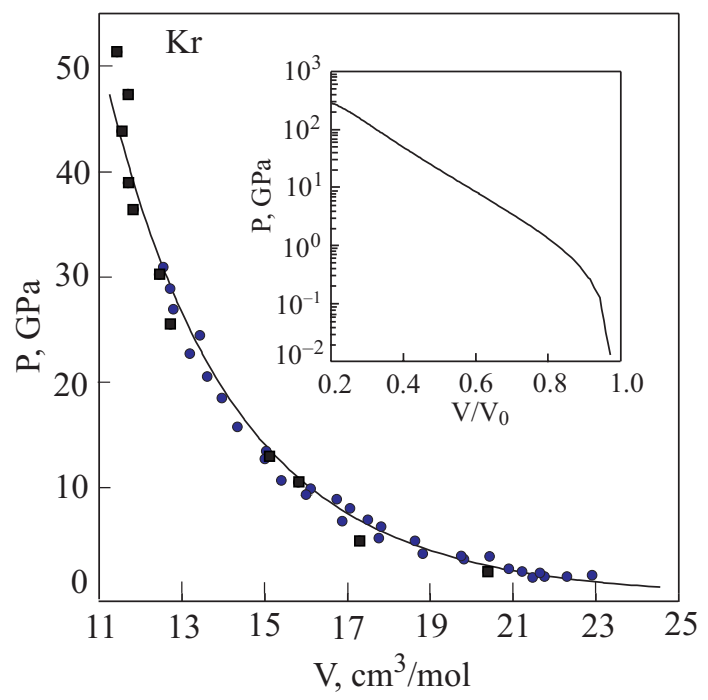

Fig. 4. The calculated $T=0 \mathrm{~K}$ equation of state for solid $\mathrm{Kr}$. Solid symbols are experimental data of Polian et al. [38,39] (circules) and of Aleksandrov et al. [40] (squares). The inset shows the zero-temperature pressures on the logarithmic scale extended to the terapascal range as a function of compression. 
tion, but our results are in markedly better agreement with experiment. Additional studies are needed to answer the question: whether this difference is connected with the fact that we used the real many-body potential whereas Kim et al. used the effective two-body potential or resulted from other details of calculations.

In comparison to other RGS, there are numerous $P-V$ measurements of solid Xe. In early piston-displacement low-temperature measurements of Anderson and Swenson [30] the pressure range up to $2.5 \mathrm{GPa}$ was investigated, and Syassen and Holzapfel [42] in tungsten- and boron-carbide anvil studies extended $P-V$ measurements to $11 \mathrm{GPa}$. Schiferl et al. [43], Asaumi [21], Zisman et al. [44] brought EOS measurements up to 23, 32 and $53 \mathrm{GPa}$, respectively. Jephcoat et al. [45], and Reichlin et al. [47] extended EOS experiments to 137 and $172 \mathrm{GPa}$, respectively. The highest volume compression reached in experiment is 3.55 , the same limiting value which was attained in experiments with $\mathrm{Ne}$. Jephcoat et al. found that xenon transforms at $14 \mathrm{GPa}$ from fcc to a still unidentified intermediate structure that transforms to hcp at $75 \mathrm{GPa}$. According to optical studies, insulator-to-metal transition in $\mathrm{Xe}$ occurs at pressures $132 \mathrm{GPa}$ or $150 \mathrm{GPa}$ according to Ref. 46 or Ref. 47 respectively.

The calculated $T=0$ dependence of $P(V)$ for solid xenon is shown in Fig. 5 along with the experimental results. In our calculations we used slightly modified parameters $A$ and $\alpha_{\text {tr }}$ of the short-range three-body potential (see Table 2) compared to that from Ref. 7 ( $A=$ $=1964.08$ a. u., $\alpha_{\text {tr }}=0.75249$ a.u.). Agreement between theory and experiment is excellent for the whole pressure range. Our results extended for the terapascal range are given in the Inset in Fig. 5.

The EOS of solid xenon was calculated by Loubeyre [7] and Kim et al. [8]. The comparison of our results with that from Refs. 7 and 8 exactly repeats the situation which was analyzed for solid $\mathrm{Kr}$.

\subsection{Comparison of $P(V)$ relations}

The comparison of $P(V)$ relations is usually performed between the reduced pressure $P / B_{0}\left(V_{0}\right)$ and relative volume change $V / V_{0}$, where $V_{0}$ and $B_{0}\left(V_{0}\right)$ are the $T=0, P=0$ volume and bulk modulus, respectively. At low pressures, the reduced $T=0 \mathrm{EOS} P / B_{0}\left(V_{0}\right)=f\left(V / V_{0}\right)$ are identical to a high accuracy for the three heaviest rare gases and are only slightly different for solid $\mathrm{Ne}$ [48]. At higher pressures this relation between the reduced pressures and volumes does not hold and we compared the calculated $T=0$ EOS for solid $\mathrm{He}, \mathrm{Ne}, \mathrm{Ar}, \mathrm{Kr}$, and $\mathrm{Xe}$ as a function of the relative compression $V / V_{0}$ (Fig. 6). As can be seen, much higher pressure is needed in the heavier rare gases compared with He to reach a given relative compression, which is due to the repulsive effect of the core electrons [37]. The differences between the heavier rare gases are not so large, with the

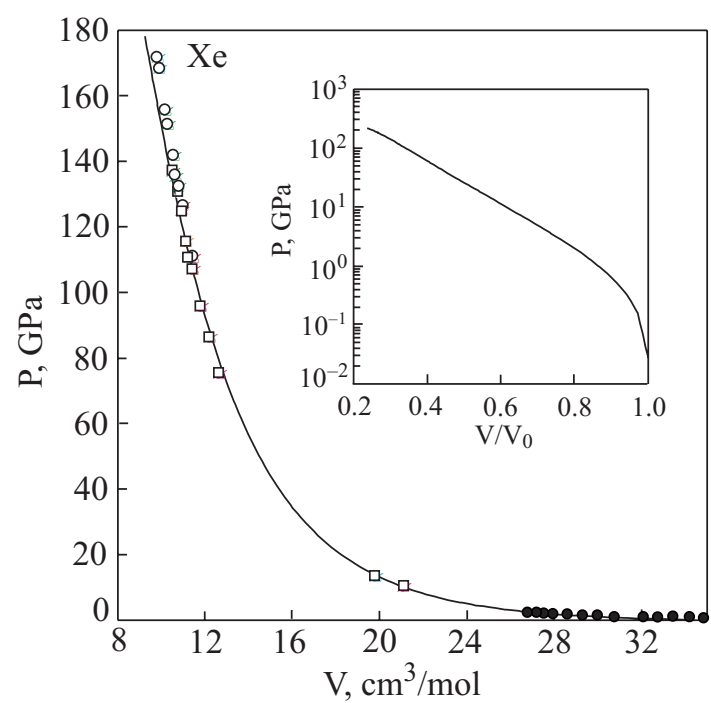

Fig. 5. The calculated $T=0 \mathrm{~K}$ equation of state for solid Xe. Solid circules are low-pressure experimental data from Ref. 30. High-pressure data: open squares Ref. 45; open circules Ref. 47. The inset shows the calculated zero-temperature pressures on the logaritmic scale extended to the terapascal range as a function of compression.

curves for $\mathrm{Ar}$ and $\mathrm{Kr}$ near coinside, which means that the latter lies anomalously low. Moreover, at higher compressions $\left(V / V_{0} \approx 0.35\right)$, the curve for $\mathrm{Ar}$ crosses that for $\mathrm{Kr}$ and at higher compressions lie above the $\mathrm{Kr}$ curve.

This anomaly is a consequence of the fact that the three-body exchange forces and the negative contribution to the pressure along with it increase with volume compression $V / V_{0}$ faster for $\mathrm{Kr}$ than for $\mathrm{Ar}$ (Fig. 7). Analyzing the relative magnitude of the three-body contribution to the ground-state energy with respect to the two-body interaction as a function of $V_{0} / V$ we found that this value is non-monotonic in the sequence $\mathrm{Ne}-\mathrm{Ar}-\mathrm{Kr}-\mathrm{Xe}$. In the range of compressions two- to five-fold the curve for $\mathrm{Kr}$

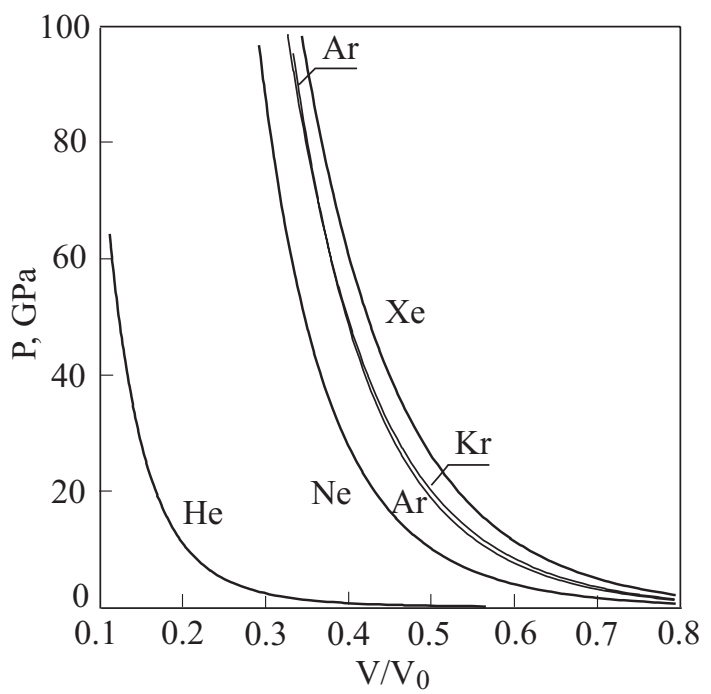

Fig. 6. The calculated $T=0 \mathrm{~K}$ equations of state for solid $\mathrm{He}, \mathrm{Ne}$, $\mathrm{Ar}, \mathrm{Kr}$, and $\mathrm{Xe}$ as a function of the relative compression $V / V_{0}$. 
lies higher than those for other RGS. Surprisingly, Xe has the lowest value at the two-fold compressions but its curve goes steeper and intersects susccessively the curves for $\mathrm{Ne}$ and Ar. The intersection point with the $\mathrm{Kr}$ curve lies outside the five-fold compression where the adopted approximation becomes inappropriate.

\section{Conclusions}

The EOS of rare gas solids ( $\mathrm{He}, \mathrm{Ne}, \mathrm{Ar}, \mathrm{Kr}$, and $\mathrm{Xe}$ ) are calculated in the experimentally studied ranges of pressures taking into account two- and three- body interatomic forces. In the capacity of the two-body potentials we used potentials developed for the whole group of substances by Aziz and collaborations [12-15]. They are derived from two-body properties and represent pair interaction between two isolated atoms. The solid-phase intermolecular potentials which we used to describe the EOS in solids up to very high volume compressions contained the long-range Axilrod-Teller three-body term and short-range three-body exchange interaction. The latter we used in the Slater-Kirkwood form and treated its energy-scale and length-scale parameters as adjusted parameters of theory. The long-range three-body term is repulsive and the resulted positive correction to the pressure is essential at small pressures, while the short-range three-body term is attractive and contributes the negative correction to the pressure, which means that the three-body exchange interaction softens the two-body repulsion at high pressure. The calculated $T=0 \mathrm{~K}$ EOS are in excellent agreement with experiment in the whole range of pressures for all RGS. The calculated EOS for $\mathrm{Ar}, \mathrm{Kr}$, and Xe extended for the terapascal range exhibit inflection points where the isothermal bulk moduli have non-physical maxima indicating that the limitation of the many-body forces by the three-body interactions becomes invalid. These points lie at pressures 250 , 200 , and $175 \mathrm{GPa}$ (the volume compressions 4.76, 4.08, and

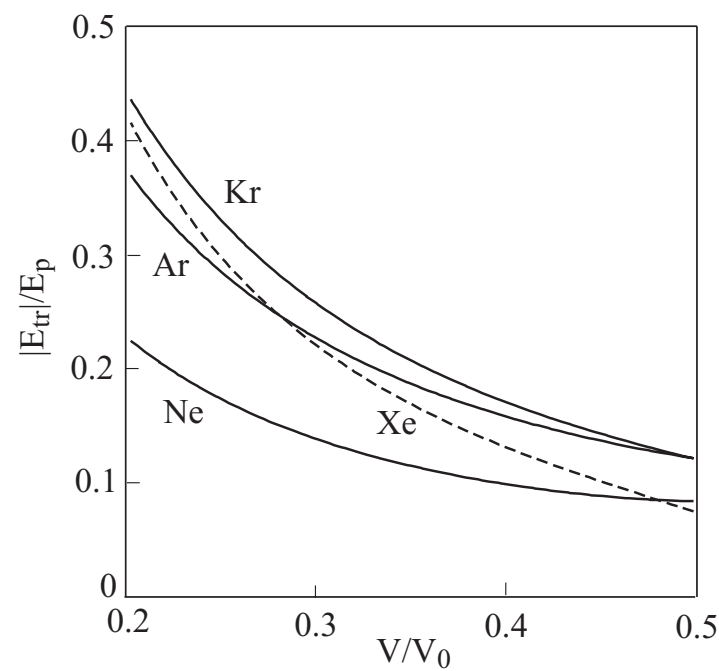

Fig. 7. The ratio between contributions of the triple $E_{\mathrm{tr}}$ and pair forces $E_{p}$ to the ground state energy $E_{g s}$ of $\mathrm{Ne}, \mathrm{Ar}, \mathrm{Kr}$, and $\mathrm{Xe}$ as a function of the relative volume compression $V / V_{0}$.
3.57) for $\mathrm{Ar}, \mathrm{Kr}$, and $\mathrm{Xe}$, respectively. No such points were found for $\mathrm{He}$ and $\mathrm{Ne}$. Let us note that this level of compressions is reached in experiment for $\mathrm{Ne}$ and Xe but, for unknown reasons, the reached compressions for $\mathrm{Ar}$ and $\mathrm{Kr}$ are markedly smaller with the least for $\mathrm{Kr}$ (less than 2.4).

The zero-point energy and respectively $T=0 \mathrm{~K}$ EOS for the heavier EOS ( $\mathrm{Ne}, \mathrm{Ar}, \mathrm{Kr}$, and $\mathrm{Xe}$ ) starting from relatively small compressions (1.5-fold and lower for the heaviest RGS) is dominated by the static lattice contributions. At the highest compression reached (3.5-fold) the zero-point contribution to the ground state of $\mathrm{Ne}$ is about $10 \%$. In the case of solid $\mathrm{He}$, at compressions approximately 3 -fold and smaller the ground-state is dominated by the zero-point oscillations. The static lattice contributions becomes dominating starting approximately from 6-fold compressions. At the highest pressure reached (about $60 \mathrm{GPa}$ ) the zero-point contribution to the ground state of $\mathrm{He}$ is still about $25 \%$.

The relative magnitude of the three-body contribution to the ground energy with respect to the two-body interaction as a function of $V_{0} / V$ was found to be non-monotonic in the sequence $\mathrm{Ne}-\mathrm{Ar}-\mathrm{Kr}-\mathrm{Xe}$. In the range of compressions two- to five-fold the highest value has Kr. Surprisingly, Xe at the two-fold compression has the least value but it increases with compression faster than others. This anomally high three-body contribution where dominates the attractive short-range exchange forces results in so large negative pressure that the EOS for $\mathrm{Kr}$ and $\mathrm{Ar}$ as a function of compression near coincide with that for $\mathrm{Ar}$ even intersecting the $\mathrm{Kr}$ curve at the compressions higher approximately 3.5 .

1. S.M. Tretyak, T.N. Antsygina, and Yu.A. Freiman, Fiz. Nizk. Temp. 32, 1408 (2006) [Low. Temp. Phys. 32, 1072 (2006)].

2. C.S. Zha, H.K. Mao, and R.J. Hemley, Phys. Rev. B70, 174107 (2004).

3. Yu.A. Freiman, S.M. Tretyak, A. Jeżowski, and R.J. Hemley, J. Low Temp. Phys. 126, 703 (2002); ibid. 122, 537 (2001).

4. M. Neumann and M. Zoppi, Phys. Rev. 62, 41 (2000).

5. J.A. Barker, in: Simple Molecular Systems at Very High Density, A. Polian, P. Loubeyre, and N. Boccara (eds.), Plenum Press, New York (1989).

6. P. Loubeyre, Phys. Rev. Lett. 58, 1857 (1987).

7. P. Loubeyre, Phys. Rev. B37, 5432 (1988).

8. J.H. Kim, T. Ree, and F.H. Ree, J. Chem. Phys. 91, 3133 (1989).

9. J. Barker, in: Rare Gas Solids, M.L. Klein and J.A. Venables (eds.), Academic Press, New York (1976), v. 1, ch. 6.

10. I.F. Silvera and V.V. Goldman, J. Chem. Phys. 69, 4209 (1978).

11. R.J. Hemley, H.K. Mao, L.W. Finger, A.P. Jephcoat, R.M. Hazen, and C.S. Zha, Phys. Rev. 42, 6458 (1990).

12. R.A. Aziz, V.P.S. Nain, J.S. Carley, W.L. Taylor, and G.T. McConville, J. Chem. Phys. 70, 4330 (1979).

13. R.A. Aziz and M.J. Slaman, Chem. Phys. 130, 187 (1989). 
14. R.A. Aziz and M.J. Slaman, Molec. Phys. 58, 679 (1986).

15. R.A. Aziz and M.J. Slaman, Molec. Phys. 57, 825 (1986).

16. M. Bulski and G. Chalasinski, Chem. Phys. Lett 89, 450 (1982).

17. L.W. Bruch and I.J. McGee, J. Chem. Phys. 59, 409 (1973).

18. M.B. Doran and I.J. Zucker, J. Phys. C4, 307 (1971).

19. A.C. Holt and M. Ross, Phys. Rev. B1, 2700 (1970).

20. R.J. Hemley, C.S. Zha, A.P. Jephcoat, H.K. Mao, L.W. Finger, and D.E. Cox, Phys. Rev. B39, 11820 (1989).

21. K. Asaumi, Phys. Rev. B29, 7026 (1984).

22. M. Ross, H.K. Mao, P.M. Bell, and J.A. Xu, J. Chem. Phys. 85, 1028 (1986).

23. J.W. Stewart, Phys. Rev. 129, 1950 (1963).

24. A. Driessen, E. van der Poll, and I.F. Silvera, Phys. Rev. B33, 3269 (1986).

25. R.L. Mills, D.H. Liebenberg, and J.C. Bronson, Phys. Rev. B21, 5137 (1980).

26. W.J. Nellis, N.C. Holmes, A.C. Mitchell, R.J. Trainor, G. K. Governo, M. Ross, and D.A. Young, Phys. Rev. Lett. 53, 1248 (1984).

27. H.K. Mao, R.J. Hemley, Y. Wu, A.P. Jephcoat, L. W. Finger, C.S. Zha, and W.A. Basset, Phys. Rev. Lett. 60, 2649 (1988).

28. P. Loubeyre, R. LeToullec, P. Loubeyre, and J.P. Pinceaux, Phys. Rev. B40, 2368 (1989).

29. P. Loubeyre, R. LeToullec, J.P. Pinceaux, H.K. Mao, J. Hu, and R. J. Hemley, Phys. Rev. Lett. 71, 2272 (1993).

30. M.S. Anderson and C.A. Swenson, J. Phys. Chem. Solids 10, 145 (1975).

31. R.M. Hazen, H.K. Mao, L.W. Finger, and P.M. Bell, Carnegie Inst. Washington Yearb. 79, 348 (1980).

32. L.W. Finger, R.M. Hazen, G. Zou, H.K. Mao, and P.M. Bell, Appl. Phys. Lett. 39, 348 (1980).
33. R.J. Hemley and H.K. Mao, J. Low Temp. Phys. 122, 331 (2001).

34. V.N. Zharkov and V.P. Trubitsyn, Physics of Planetary Interiors, Pachart, Tucson, Arizona (1978).

35. J. Hama, Phys. Lett. A105, 303 (1986).

36. J.C. Boettger and S.B. Trickey, Phys. Rev. B29, 6425 (1984).

37. J.C. Boettger, Phys. Rev. B33, 6788 (1986).

38. A. Polian, J.M. Besson, M. Grimsditch, and W.A. Grosshans, Phys. Rev. B39, 1132 (1989).

39. A. Polian, J.P. Itie, E. Dartyge, A. Fontaine, and G. Tourillon, Phys. Rev. B39, 3369 (1989).

40. I.V. Aleksandrov, A.N. Zisman, and S.M. Stishov, Sov. Phys. JETP 65, 371 (1987).

41. J.A. Barker, J. Stat. Phys. 52, 1359 (1988).

42. K. Syassen and W.B. Holzapfel, Phys. Rev. B18, 5826 (1978).

43. D. Schiferl, R.L. Mills, and L.E. Trimmer, Solid State Commun. 46, 783 (1983).

44. A.N. Zisman, I.V. Aleksandrov, and S.M. Stishov, Phys. Rev. B32, 484 (1985).

45. A.P. Jephcoat, H.K. Mao, L.W. Finger, D.E. Cox, R.J. Hemley, and C.S. Zha, Phys. Rev. Lett. B59, 2670 (1987).

46. K.A. Goettel, J.H. Eggert, and I.F. Silvera, Phys. Rev. Lett. 62, 665 (1989).

47. R. Reichlin, K.E. Brister, A.K. McMahan,, M. Ross, S. Martin, Y.K. Vohra, and A.L. Ruoff, Phys. Rev. Lett. 62, 669 (1989).

48. C.A. Swenson, in: Rare Gas Solids, M.L. Klein and J.A. Venables (eds.), Academic, New York (1977), v. II, ch. 13, p. 877. 